\title{
Stresses and Strains Developed by the Reflection of Seismic Waves at a Free Surface
}

John R. Banister, D. Maxwell Ellett, Clarence R. Mehl, Frank F. Dean

Prepared by Sandia Laboratories, Albuquerque, New Mexico 87185

and Livermore, California 94550 for the United States Department

of Energy under Contract AT (29-1)-789

Printed July 1978 


\section{DISCLAIMER}

This report was prepared as an account of work sponsored by an agency of the United States Government. Neither the United States Government nor any agency Thereof, nor any of their employees, makes any warranty, express or implied, or assumes any legal liability or responsibility for the accuracy, completeness, or usefulness of any information, apparatus, product, or process disclosed, or represents that its use would not infringe privately owned rights. Reference herein to any specific commercial product, process, or service by trade name, trademark, manufacturer, or otherwise does not necessarily constitute or imply its endorsement, recommendation, or favoring by the United States Government or any agency thereof. The views and opinions of authors expressed herein do not necessarily state or reflect those of the United States Government or any agency thereof. 


\section{DISCLAIMER}

Portions of this document may be illegible in electronic image products. Images are produced from the best available original document. 
Issued by Sandia Laboratories, operated for the United States Department of Energy by Sandia Corporation.

\section{NOTICE}

This report was prepared as an account of work sponsored by the United States Government. Neither the United States nor the Department of Energy, nor any of their employees, nor any of their contractors, subcontractors, or their employees, makes any warranty, express or implied, or assumes any legal liability or responsibility for the accuracy, completeness or usefulness of any information, apparatus, product or process disclosed, or represents that its use would not infringe privately owned rights.

Printed in the United States of America

Available from

National Technical Information Service

U. S. Department of Commerce

5285 Port Royal Road

Springfield, VA 22161

Price: Printed Copy $\$ 4.50$; Microfiche $\$ 3.00$ 


\section{PAGES 1 to 2 WERE INTENTIONALLY LEFT BLANK}


SAND77-0673

Unlimited Release

Printed July 1978

STRESSES AND STRAINS DEVELOPED BY THE REF LECTION OF SEISMIC WAVES AT A FREE SURFACE

John R. Banister

Geosciences Department 1150

D. Maxwell Ellett

Applied Geophysics Division 1152

Clarence R. Mehl

Field Experiments Division 1111

Frank F. Dean

Field Experiments Division 1111

Sandia Laboratories

Albuquerque, New Mexico 87185

\section{ABSTRACT}

Exact and approximate equations have been derived for the stresses and strains beneath a free surface when an incoming longitudinal wave and an incoming shear wave reflect from the surface. Results of the approximate solution for depths much less than the wave length of the incoming wave are given in tabular form and are graphed for Poisson's ratios of $0.25,0.3$, and 0.333 . The results should be of use in categorizing the magnitude of near-surface stresses and strains resulting from seismic waves produced by deeply buried explosives or earthquakes.

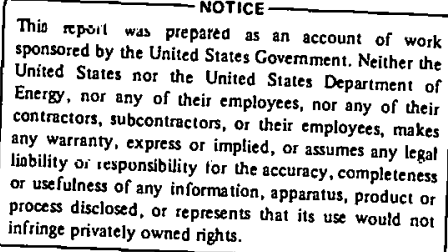




\section{STRESSES AND STRAINS DEVELOPED BY THE REFLECTION OF SEISMIC WAVES AT A FREE SURFACE}

It is important to understand the behavior and magnitude of the stresses and straing which occur when seismic waves from earthquakes or underground nuclear explosions are.reflected at the earth's surface. Such knowledge is especially useful for examining the regions close to the surface where the disturbances might affect buildings or cause such phenomena as soil liquefaction. With this purpose in mind we have made the following study. Although the direction and magnitude of an incident seismic wave is rarely known exactly, we hope that a general understanding of the effects and the trends of the results will help in explaining phenomena which are otherwise difficult to interpret.

Extensive studies of the reflection of a seismic longitudinal or shear wave at a free surface can be found in various forms in many. standard texts. $1,2,3,4$ The derivations of the amplitude coefficients of the reflected waves or the expresstons for the stress are of ten used as exercises for the student. However, there are no tables or graphs in one place that give the results collected for the amplitude coefficients, stresses, and strains for a variety of angles of incidence and Poisson's ratios. In this note, we will look particularly into the problem for both incoming longitudinal and shear waves in the region close to the surface (e.g. where the depth is small compared to the length of the seismic wave).

We shall treat the problem in two parts. In the first part we shall consider the problem of a longitudinal wave reflecting at a free boundary. In the second part, we shall treat the problem of a shear wave reflecting at a free boundary. The second part will be further subdivided into two solutions which depend on whether or not a secondary longitudinal wave may be generated at the free surface.

Given an incident longitudinal wave approaching the surface as shown in Figure 1 , we may agsume the particle displacement of the wave is a sinusoidal function, and write this function as

$$
\Phi_{1}=\operatorname{Re}\left\{A_{1} e^{\left[i\left(p t+f_{1} x+g_{1} y\right)\right]}\right\}
$$

where

$$
\begin{aligned}
& A_{1}=\text { displac ement amplitude of the longitudinal wave (cm.) } \\
& p=\text { angular frequency, } \mathrm{rad} / \mathrm{sec} \\
& t=\text { time }(\mathrm{sec})
\end{aligned}
$$




$$
\begin{aligned}
& f_{1}=\frac{p \cos \alpha_{1}}{c_{1}} \\
& c_{1}=\text { velocity of body waves in the medium } \\
& x=\text { depth } \\
& g_{1}=\frac{p \text { sin } \alpha_{1}}{c_{1}} \\
& a_{1}=\text { angle of incidence } \\
& y=\text { distance from origin parallel to the surface }
\end{aligned}
$$

When such a wave strikes the reflecting surface, a longitudinal wave $\Phi_{2}$ of amplitude $A_{2}$ is reflected making an angle of $\alpha_{2}$ with the normal and shear wave $\Phi_{3}$ of amplitude $A_{3}$ reflected at an angle of $\beta$ from the normal. The assumed form for the functions describing the waves is

$$
\begin{aligned}
& \Phi_{2}=\operatorname{Re}\left\{A_{2} e^{\left.\left.i\left(p t-f_{2} x+g_{2} y+\delta_{1}\right)\right]\right\}}\right. \\
& \Phi_{3}=\operatorname{Re}\left\{A_{3} e^{\left.\left.i\left(p t-f_{3} x+g_{3} y+\delta_{2}\right)\right]\right\}}\right. \\
& f_{2}=\frac{p \cos \alpha_{2}}{c_{1}} \quad g_{3}=\frac{p \sin \alpha_{2}}{\theta_{1}} \\
& f_{3}=\frac{p \cos B}{c_{2}} \quad g_{3}=\frac{p \sin B}{c_{2}} \\
& c_{2}=\text { velocity of shear waves in the medium }
\end{aligned}
$$

where $\delta_{1}$ and $\delta_{2}$ are phase angles which can be shown equal to zero.

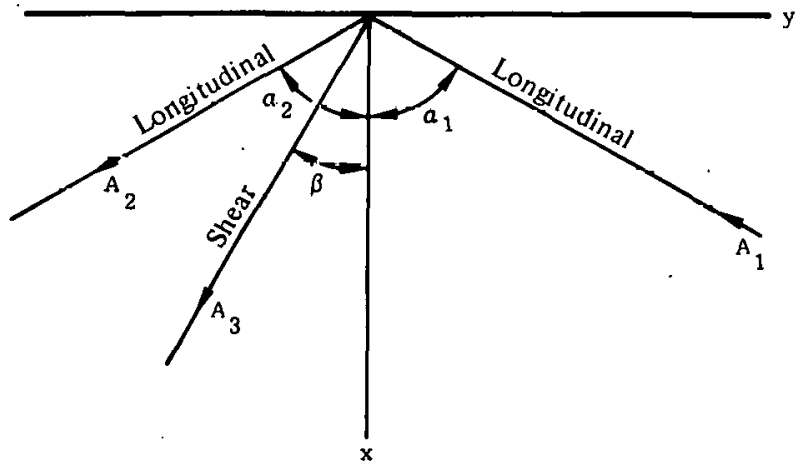

Figure 1

Incident Longitudinal Wave, Reflected Longitudinal and Shear Wave 
It can also be shown that $\alpha_{1}=\alpha_{2}$; therefore, we may drop the subscripts. The relation between $\alpha$ and $\beta$ is a function of Poisson's ratio such that:

$$
\frac{\sin \alpha}{\sin \beta}=\frac{c_{1}}{c_{2}}=\sqrt{\frac{2-2 \nu}{1-2 \nu}}=\sqrt{\frac{\lambda+2 \mu}{\mu}}=\sqrt{2+\frac{\lambda}{\mu}},
$$

where

$$
\begin{aligned}
& c_{1}=\sqrt{\frac{\lambda+2 \mu}{\rho}} \\
& c_{2}=\sqrt{\frac{\mu}{\rho}} \\
& \nu=\text { Poisson's ratio of medium } \\
& \lambda_{\mu}=\text { Lamé's Constants } \\
& \rho=\text { density of medium . }
\end{aligned}
$$

Because the shear stress $\sigma_{y x}$ at a free surface, must be 0 , one equation relates the amplitude of the three waves:

$$
2\left(A_{1}-A_{2}\right) \cos \alpha \sin B-A_{3} \cos 2 B=0
$$

At a free boundary, the stress in the $x$ direction $\sigma_{x x}$ must disappear. This leads to a second relation between the amplitude of the three waves:

$$
\left(A_{1}+A_{2}\right) \cos 2 B \sin \alpha-A_{3} \sin \beta \sin 2 \beta=0
$$

Dividing both of the last two equations by $A_{1}$, we have two equations to solve for $A_{2} / A_{1}$, and $A_{3} / A_{1}$, giving

$$
\begin{aligned}
& \frac{A_{2}}{A_{1}}=\frac{\left(\frac{C_{2}}{c_{1}}\right)^{2} \sin 2 \alpha \sin 2 \beta-\cos ^{2} 2 \beta}{R} \\
& \frac{A_{3}}{A_{1}}=\frac{2}{R}\left(\frac{C_{2}}{C_{1}}\right) \sin 2 \alpha \cos 2 \beta
\end{aligned}
$$


where

$$
\mathrm{R}=\left(\frac{\mathrm{c}_{2}}{\mathrm{c}_{1}}\right)^{2} \sin 2 \alpha \sin 2 \beta+\cos ^{2} 2 \beta
$$

The values for $A_{2} / A_{1}$ and $A_{3} / A_{1}$ are given in Table $I$ and Figure 2 for several values of Poisson's ratio which are representative of the usual range of that ratio for geologic materials.

Having solved for the displacement of the reflected wave in terms the displacement of the incident wave and following Kolsky's derivation, we may solve for the value of the stresses on the $x x, y y, z z$, and $x y$ planes. These are

$$
\begin{aligned}
& \sigma_{x x}=\frac{2 p \mu A}{c_{1} R} \cos 2 \beta\left\{\sin 2 \alpha \sin 2 \beta\left(\cos f_{1} x-\cos f_{3} x\right) \cos p t\right. \\
& \left.-\left[\frac{c_{1}^{2}}{c_{2}^{2}} \cos ^{2} 2 \beta \sin f_{1} x+\sin 2 \alpha \sin 2 \beta \sin f_{3} x\right] \sin p t\right\} \\
& \sigma_{\mathrm{yy}}=\frac{2 \mathrm{p} \mu \mathrm{A}_{1}}{\mathrm{c}_{1} \mathrm{R}}\left\{\sin 2 \alpha \sin 2 \beta\left[2\left(1-\frac{\mathrm{c}_{2}^{2}}{\mathrm{c}_{1}^{2}}\right) \cos \mathrm{f}_{1} \mathrm{x}-\cos 2 \beta\left(\cos \mathrm{f}_{1} \mathrm{x}-\cos \mathrm{f}_{3} \mathrm{x}\right)\right] \cos \mathrm{pt}\right. \\
& +\cos 2 \beta\left[\cos 2 \alpha \cos 2 \beta \sin \mathrm{f}_{1} \mathrm{x}+\sin 2 \alpha \sin 2 \beta \sin \mathrm{f}_{3} \mathrm{x}\right. \\
& \left.\left.-\left(\frac{c_{1}^{2}}{c_{2}^{2}}-1\right) \cos 2 B \sin f_{1} x\right] \sin p t\right\} \\
& \sigma_{z z}=\frac{2 p \lambda A}{c_{1} R}\left[\frac{c^{2}}{c_{1}^{2}} \sin 2 \alpha \sin 2 \beta \cos f_{1} x \cos p t-\cos ^{2} 2 \beta \sin f_{1} x \sin p t\right] \\
& \sigma_{x y}=\frac{2 p \mu A}{1} c_{1} R \sin 2 a\left\{\cos ^{2} 2 \beta\left(\cos f_{1} x-\cos f_{3} x\right) \cos p t\right. \\
& \left.-\left[\frac{c_{2}^{2}}{c_{1}^{2}} \sin 2 \alpha \sin 2 \beta \sin f_{1} x+\cos ^{2} 2 \beta \sin f_{3} x\right] \sin p t\right\} \\
& \sigma_{x z}=\sigma_{y z}=0,
\end{aligned}
$$


TABLE Ia

Values of Amplitudes, Stresses and Strains for an Incoming Longitudinal Wave, Poisson's Ratio of 0.25

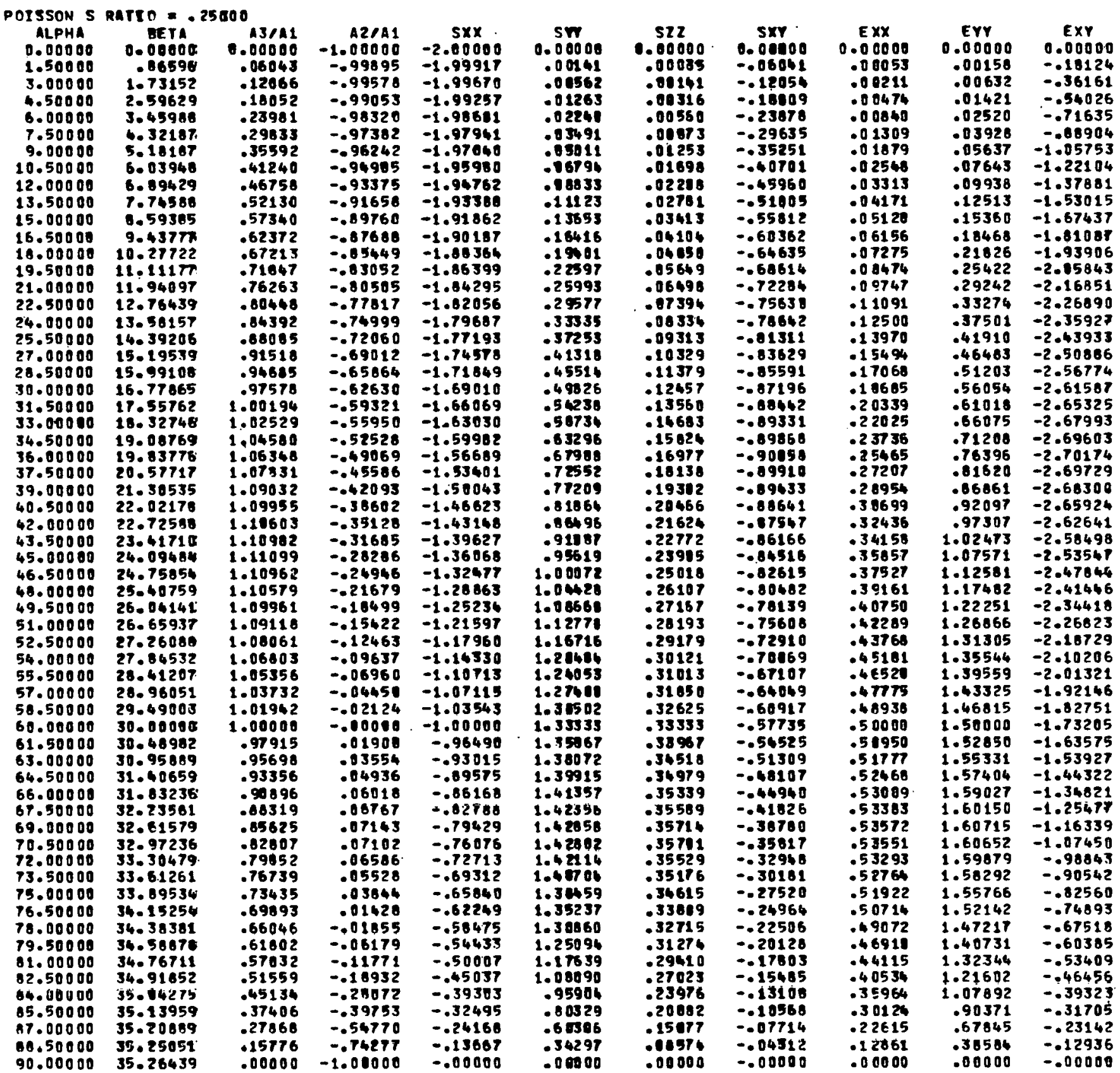


T ABLE Ib

Values of Amplitudes, Stresses and Strains for an Incoming

Longitudinal Wave, Poisson's Ratio of 0.30

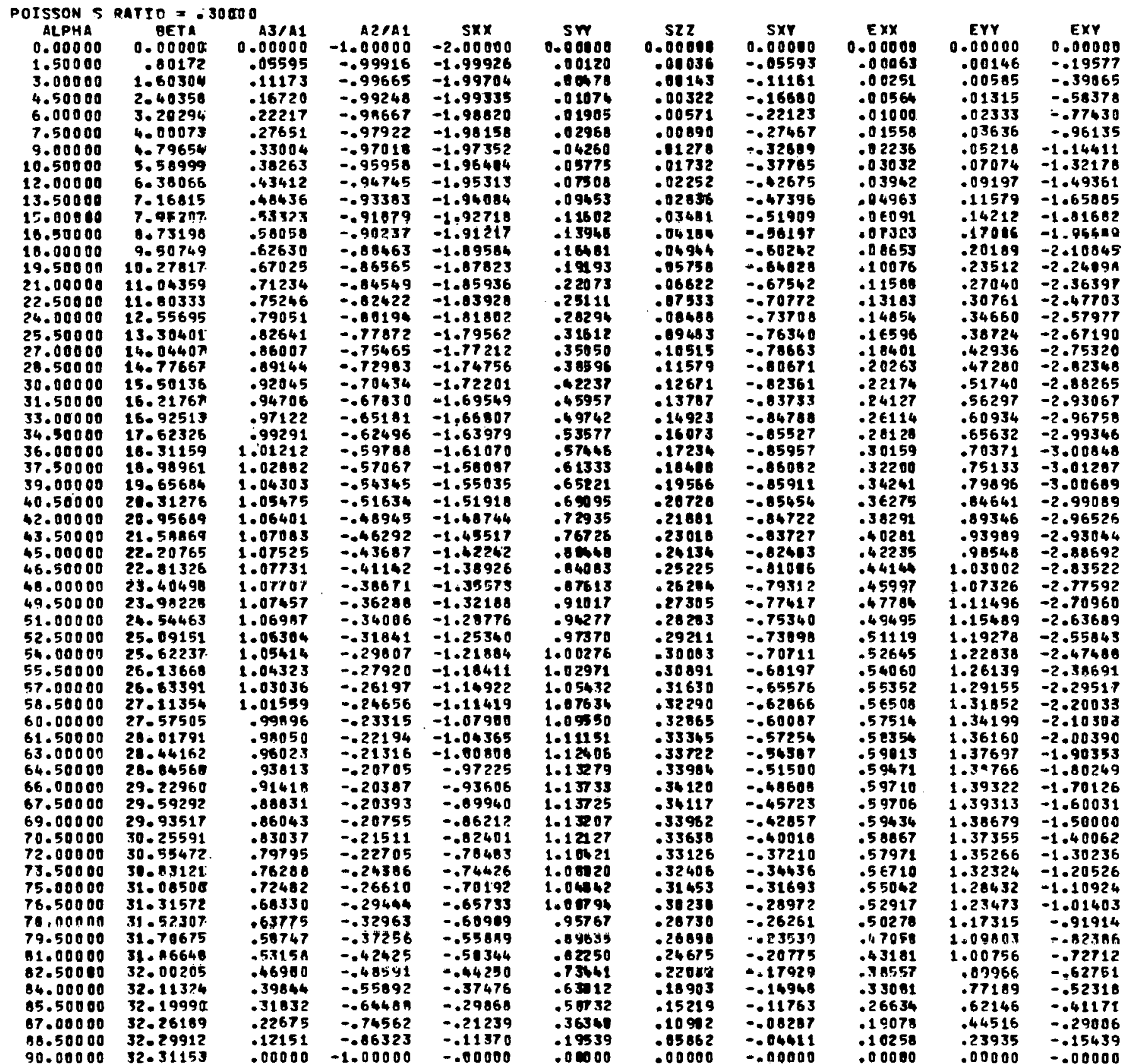


TABLE IC

Values of Amplitudes, Stresses and Strains for an Incoming

Longitudinal Wave, Poisson's Ratio of 0.333

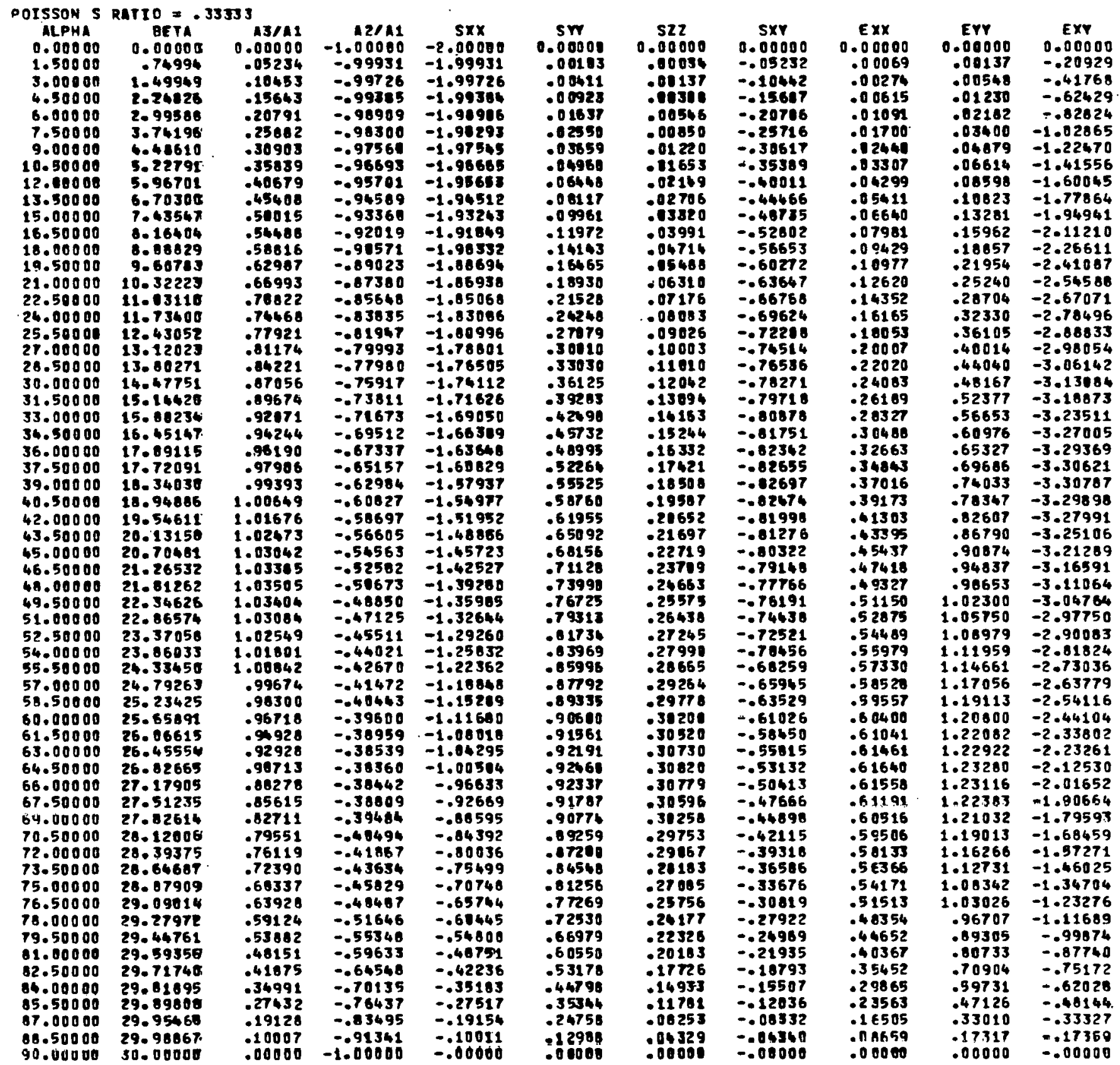




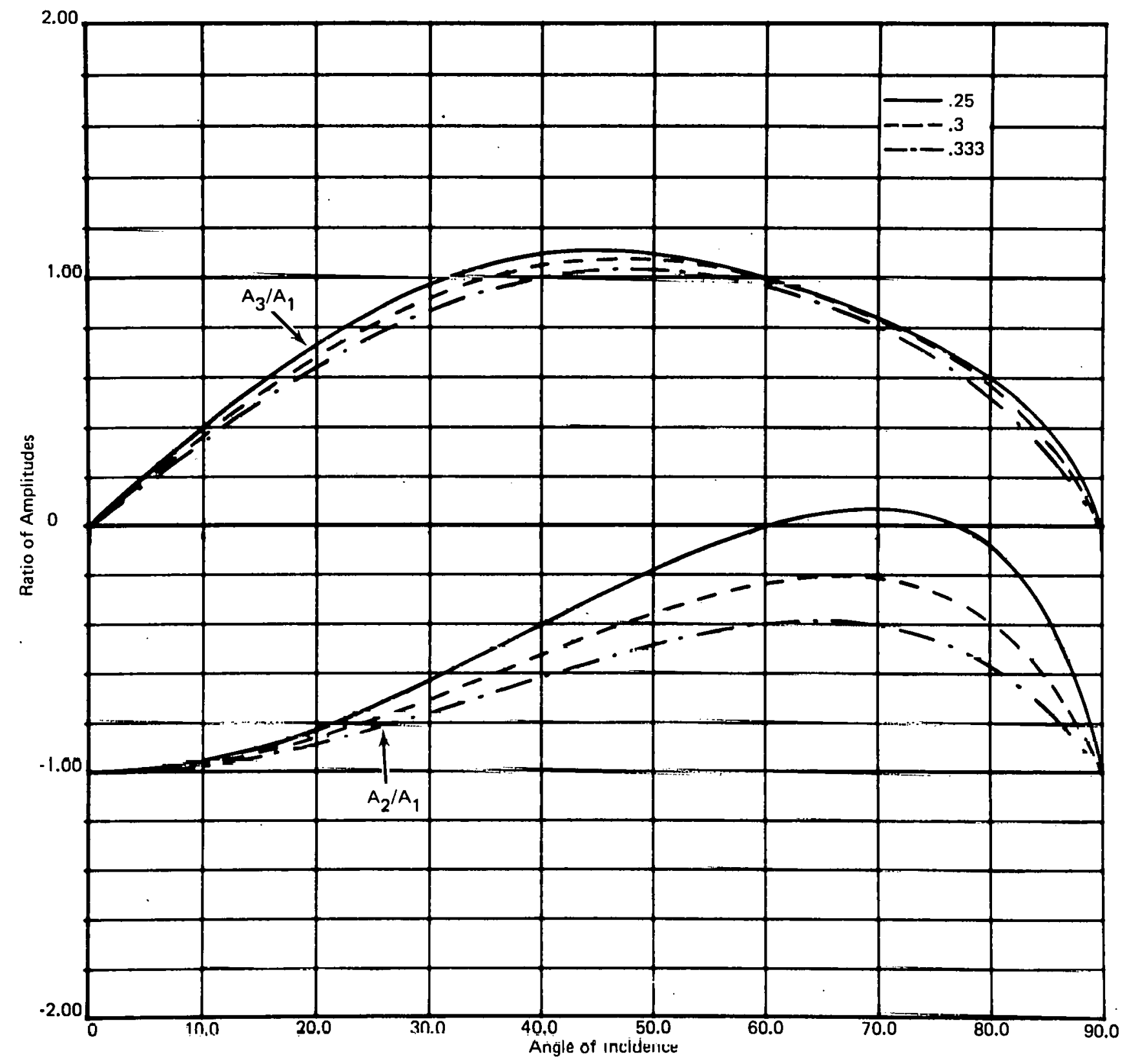

Figure 2. Plot of Ratios of Amplitudes $A_{2} / A_{1}$ and $A_{3} / A_{1}$ vs. Angle of Incidence for Poisson's Ratios of $0.25,0.30$ and 0.333 . 
These expressions are. somewhat unwieldy and are not a linear function of $x$. If we are mainly interested in the stress at depths much less than a wave length down from the surface, we may use the approximations for small $\mathbf{x}$ that

$$
\begin{aligned}
& \sin f_{1} x \approx f_{1} x \\
& \sin f_{3} x \approx f_{3} x \\
& \cos f_{1} x \approx 1 \\
& \cos f_{3} x \approx 1 .
\end{aligned}
$$

Next, rather than using the Lamé constants, we express the equations as functions of Poisson's ratio (or its equivalent $c_{1} / c_{2}$ ), the density, and the sonic velocity $c_{1}$. The rationale for this use is that both density and longitudinal velocity are relatively easy to measure in the field, and Poisson's ratio is a widely used elastic constant.

These approximations give

$$
\begin{aligned}
& \sigma_{x x}=p^{2} \rho x A_{1}\left[-\frac{2}{R} \cos 2 \beta \cos \alpha\right] \sin p t \\
& =p^{2} \rho x A_{1} S_{x x} \text { sin pt } \\
& \sigma_{y y}=p c_{1} A_{1}\left[\frac{4}{R}\left(\frac{c_{2}}{c_{1}}\right)^{2}\left(1-\left(\frac{c_{2}}{c_{1}}\right)^{2}\right) \sin 2 \alpha \sin 2 B\right] \cos p t \quad=p \rho c_{1} A_{1} s_{y y} \cdot \cos p t \\
& \sigma_{\mathrm{zz}}=p \mathrm{pc}_{1} \mathrm{~A}_{1}\left[\frac{2}{R}\left(\frac{c_{2}}{c_{1}}\right)^{2}\left(1-2\left(\frac{c_{2}}{c_{1}}\right)^{2}\right) \sin 2 \alpha \sin 2 \beta\right] \cos p t \quad \quad \nabla \rho c_{1} A_{1} s_{z z} \cos p t \\
& \sigma_{x y}=p^{2} \rho x A_{1}\left[-\frac{2}{R}\left(\frac{c_{2}}{c_{1}}\right) \sin 2 \alpha \cos \beta\left\{\left(\frac{c_{2}}{c_{1}}\right)^{4} \sin ^{2} 2 \alpha+\cos ^{2} 2 \beta\right\}\right] \sin p t \\
& =\mathrm{p}^{2} \rho \times \mathrm{A}_{1} \mathrm{~S}_{\mathrm{xy}} \sin \mathrm{pt} \text {. }
\end{aligned}
$$

To study these equations is instructive. Each equation may be divided into two parts. That part within the square brackets is nondimensional and is dependent only on Poisson's ratio and the angle that the incident wave makes with the normal to the surface. It is generally of magnitude between 0 and 2 . The other part is a coefficient which has the dimension of stress and involves the magnitude of the incoming wave $\left(A_{1}\right)$, its frequency $(p)$, the density $(\rho)$ of the medium and either the depth $(x)$ beneath the surface or the sonic velocity $\left(c_{1}\right)$ in the medium. In the case of vertical stress $\sigma_{x x}$ and shear stress $\sigma_{y x}$, the coefficient is composed of the particle acceleration of the incoming wave $\left(\mathrm{p}^{2} \mathrm{~A}_{1}\right)$ times $\rho \times$ (the mass of the overburden per unit area). In the case of the horizontal stresses, $\sigma_{y y}$ and $\sigma_{z z}$, the coefficient is the product of the particle velocity in the 
medium $\left(\mathrm{pA}_{1}\right)$ times the sonic velocity of longitudinal $\left(c_{1}\right)$ waves and density $(0)$. (This second coefficient is of the same form as that describing the stress caused by sonic waves moving through a medium.)

As stated earlier, these equations hold only for depths which are small compared to the wave length. The nondimensional parts $\mathrm{S}_{\mathbf{x x}}, \mathrm{S}_{\mathrm{xy}}, \mathrm{S}_{\mathrm{yy}}$, and $\mathrm{S}_{\mathbf{z z}}$ are given in Table I for various values of Poisson's ratio for angles between 0 and $90^{\circ}$. These results are also shown in Figure 3 .

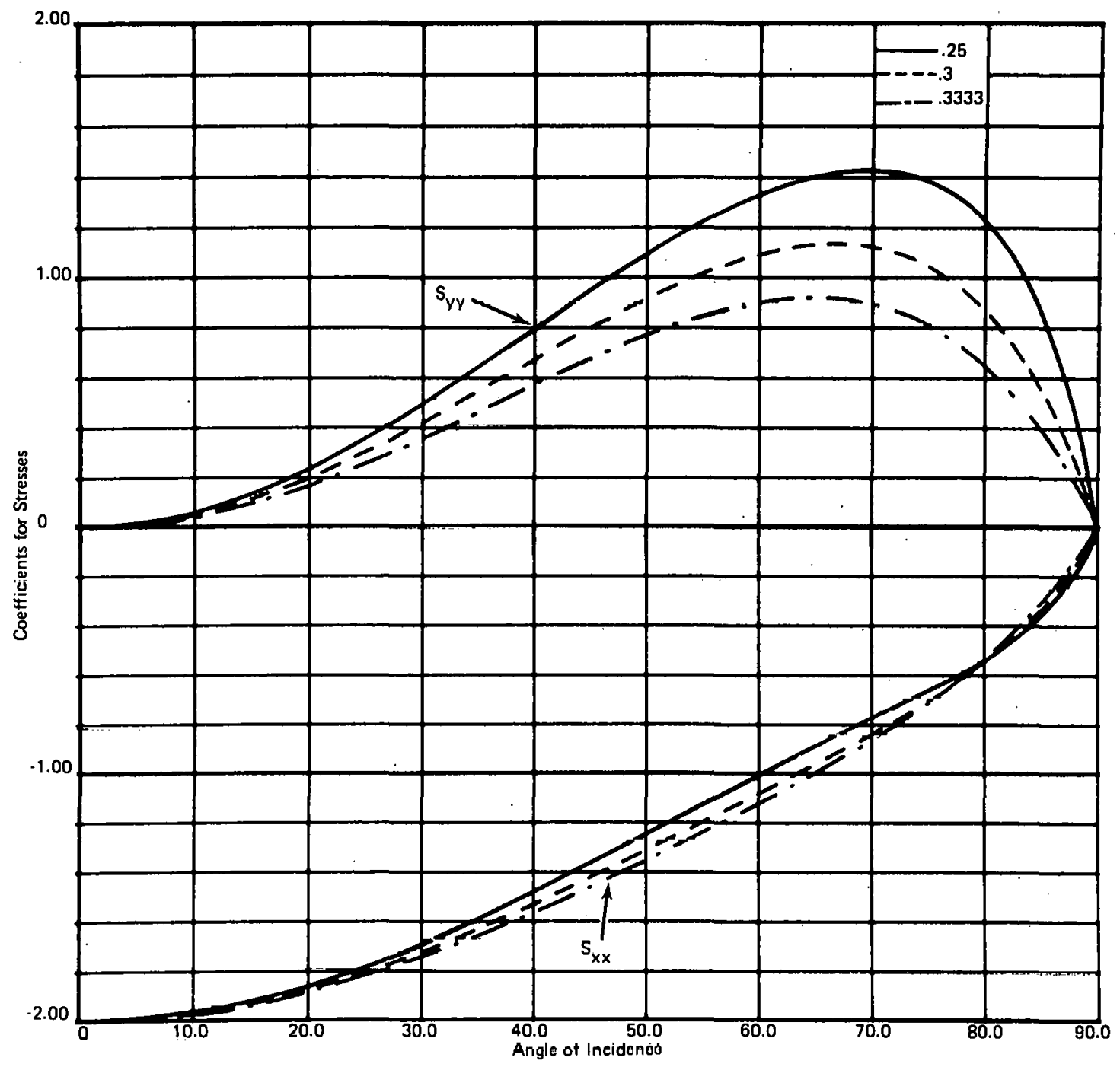

Figure 3a. Plot of the Coefficients $S_{x x}$ and $S_{y y}$ vs. Angle of Incidence for Poisson's Ratios of $0.25,0.30$ and 0.333 , Incidenl Longitudinal Wave. 


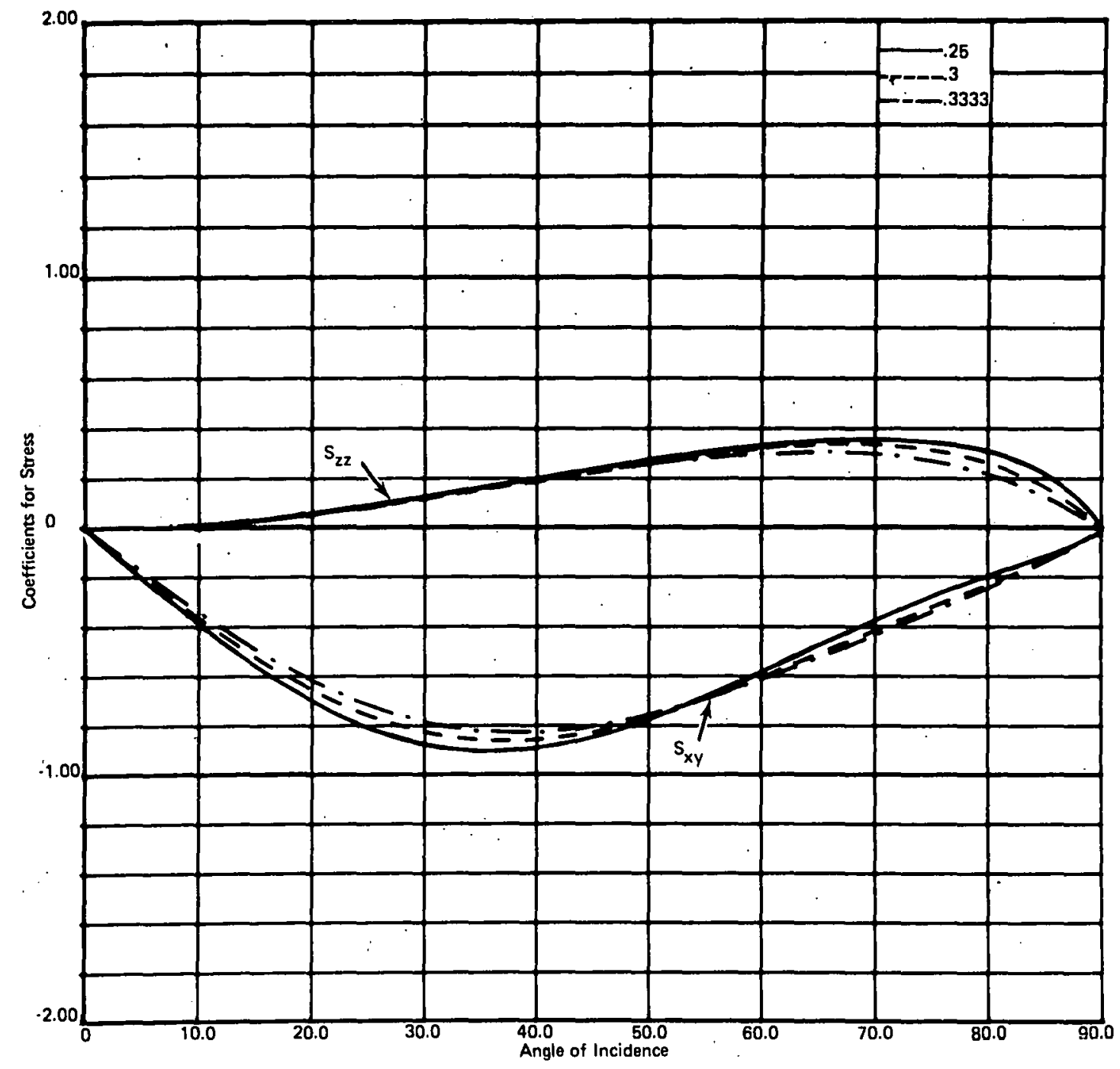

Figure 3b. Plot of the Coefficients $S_{z z}$ and $S_{x y}$ vs. Angle of Incidence for Poisson's Ratios of $0.25,0.30$ and 0.333 , Incident Longitudinal Wave.

Another item of interest is the strain $(\epsilon)$ caused by the incoming and reflected waves in both the horizontal ( $\left.\epsilon_{\mathrm{yy}}\right)$ direction and the vertical $\left(\epsilon_{\mathrm{xx}}\right)$ direction, as well as the shear strain $\epsilon_{\mathrm{xy}}{ }^{*}$ This strain may be of interest from the standpoint of measurements (e.g. those by the firm of Dames and Moore; San Francisco, Calif., on RIO BLANCO) or those strains made along a vertical line-ofsight pipe or emplacement pipe during an underground nuclear explosion. The expressions for these strains for an incident longitudinal wave such as illustrated in Figure 1 are

$$
\begin{array}{ll}
\epsilon_{\mathrm{xx}}=\frac{\mathrm{pA}_{1}}{\mathrm{c}_{1}}\left[-\frac{1}{\mathrm{R}}\left\{1-2\left(\frac{\mathrm{c}_{2}}{\mathrm{c}_{1}}\right)^{2}\right\} \sin 2 \alpha \sin 2 B\right] \cos \mathrm{pt} & =\frac{\mathrm{pA}_{1}}{\mathrm{c}_{1}} \mathrm{E}_{\mathrm{xx}} \cos \mathrm{pt} \\
\epsilon_{\mathrm{yy}}=\frac{\mathrm{pA}_{1}}{\mathrm{c}_{1}}\left[\frac{1}{\mathrm{R}} \sin 2 u \sin 2 \mathrm{~B}\right] \cos \mathrm{pt} . & =\frac{\mathrm{pA}_{1}}{\mathrm{c}_{1}} \mathrm{E}_{\mathrm{yy}} \cos \mathrm{pt}
\end{array}
$$




$$
\epsilon_{x y}=\frac{p^{2} x_{1}}{c_{1}^{2}}\left[-\frac{2}{R}\left(\frac{c_{1}}{c_{2}}\right) \sin 2 \alpha \cos \beta\left(\left(\frac{c_{2}}{c_{1}}\right)^{4} \sin ^{2} 2 \alpha+\cos ^{2} 2 \beta\right)\right] \sin p t
$$

$$
=\frac{p^{2} x A_{1}}{c_{1}^{2}} E_{x y} \sin p t
$$

These results are also shown in nondimensional form in Table $\mathrm{I}$ and in Figure 4.

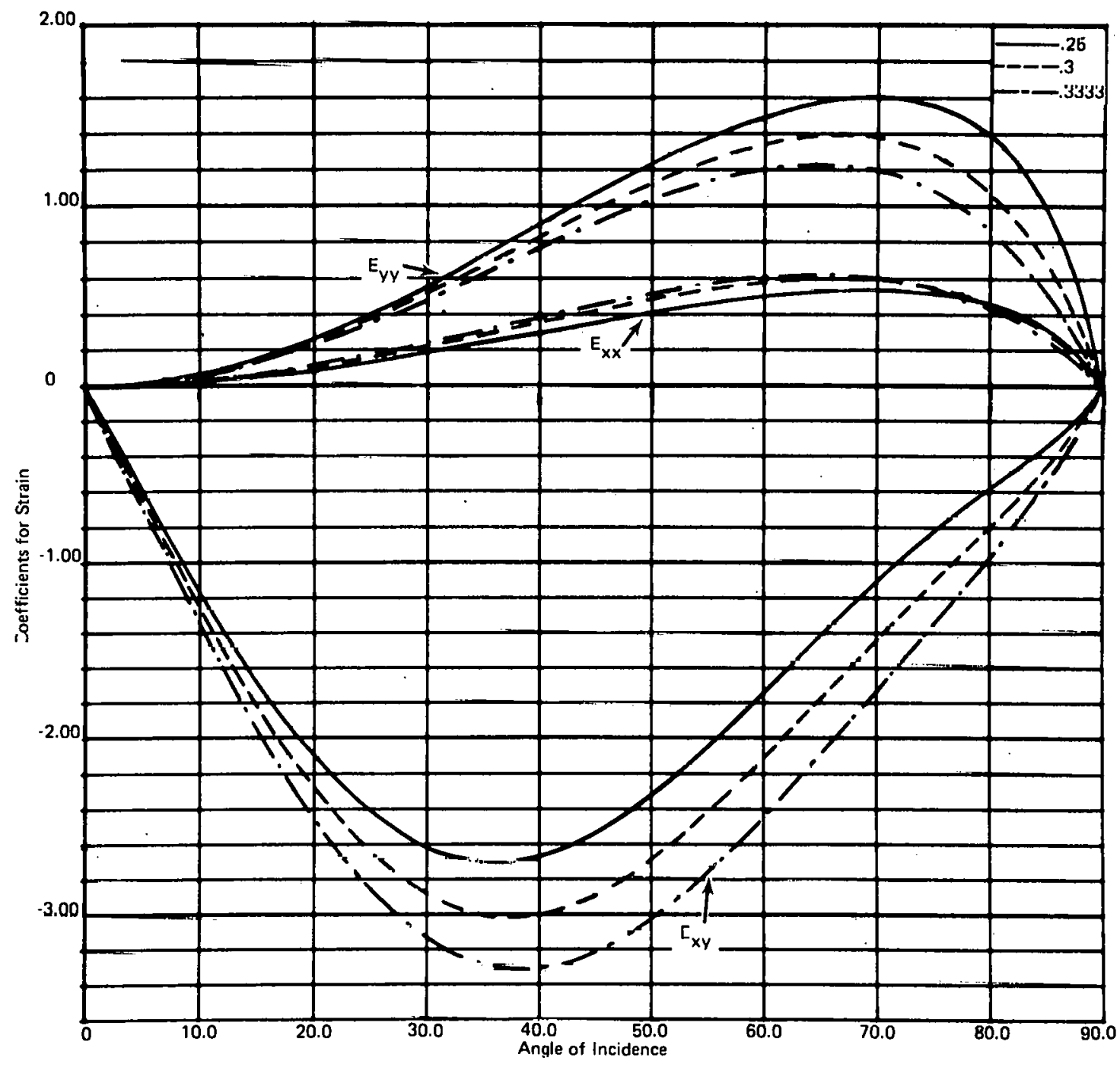

Figure 4. Plot of the Coefficients $E_{x x}, E_{y y}$ and $E_{x y}$ vs. Angle of Incidence for Poisson's Ratios of $0.25,0.30$ and 0.333 , Incident Longltudinal Wave. 
Next, we treat the subject of an incident shear wave such as is illustrated in Figure 5. First we consider a wave inclined at such an angle that there is possible both a reflected shear and a reflected longitudinal wave. This case may be treated in an analogous manner to the case of the longitudinal wave. Here we describe the incident shear wave as

$$
\theta_{1}=\operatorname{Re}\left\{B_{1} e^{\left[i\left(p t+f_{1}^{\prime} x+g_{1}^{\prime} y\right)\right]}\right\},
$$

where

$$
\begin{aligned}
& B_{1}=\text { displacement amplitude of shear wave } \\
& p=\text { angular frequency } \\
& t=\text { time (sec) } \\
& f_{1}^{\prime}=\frac{p \cos \beta}{c_{2}} \quad f_{2}^{\prime}=\frac{p \cos \beta}{c_{2}} \\
& x=\text { depth } \quad f_{3}^{\prime}=\frac{p \cos \alpha}{c_{1}} \\
& g_{1}^{\prime}=\frac{p \sin \beta}{c_{2}} \quad g_{2}^{\prime}=\frac{p \sin \beta}{c_{2}} \\
& y=\text { distance from origin parallel to the surface } \\
& \beta=\text { angle of incidence }
\end{aligned}
$$

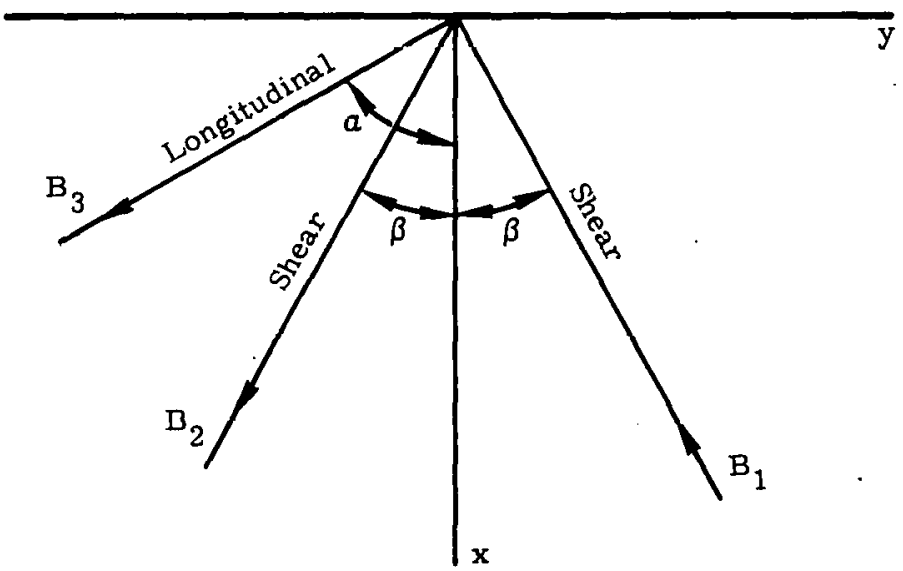

Figure 5. Incident Shear Wave, Reflected Shear and Longitudinal Waves 
In a treatment analogous to that in the previous section,

$$
\begin{aligned}
& \theta_{2}=\operatorname{Re}\left\{B_{2} e^{\left.\mid i\left(p t+f_{2}^{\prime} x+g_{2}^{\prime} y+\delta_{1}\right)\right]}\right\} \\
& \theta_{3}=\operatorname{Re}\left\{B_{3} e^{\left[i\left(p t+f_{3}^{\prime} x+g_{3}^{\prime} y+\delta_{2}\right)\right]}\right\} .
\end{aligned}
$$

It can be shown, as before, that $\delta_{1}=\delta_{2}=0$ and $g_{1}^{\prime}=g_{2}^{\prime}=g_{3}^{\prime}=g$. The same relations as were used in the case of the incident longitudinal wave and reflected longitudinal and shear waves will hold for this case, and we may use the fact that $\sigma_{x y}$ and $\sigma_{x \mathbf{x}}$ disappear at a free boundary to get expressions that permit us to calculate $B_{2} / B_{1}$ and $B_{3} / B_{1}$. Here $B_{2}$ is the amplitude of the reflected shear wave and $B_{3}$ is the amplitude of the reflected longitudinal wave.

$$
\begin{aligned}
& \frac{\mathrm{B}_{2}}{\mathrm{~B}_{1}}=\frac{\cos ^{2} 2 \beta-\left(\frac{\mathrm{C}_{2}}{\mathrm{C}_{1}}\right)^{2} \sin 2 \beta \sin 2 \alpha}{\cos ^{2} 2 \beta+\left(\frac{\mathrm{c}_{2}}{\mathrm{C}_{1}}\right)^{2} \sin 2 \beta \sin 2 \alpha}=\frac{\cos ^{2} 2 \beta-\left(\frac{\mathrm{C}_{2}}{\mathrm{C}_{1}}\right)^{2} \sin 2 \beta \sin 2 \alpha}{\mathrm{R}} \\
& \frac{\mathrm{B}_{3}}{\mathrm{~B}_{1}}=\frac{2}{\mathrm{R}} \frac{\mathrm{C}_{2}}{\mathrm{C}_{1}} \sin 2 \beta \cos 2 \beta=\frac{\frac{\mathrm{C}_{2}}{\mathrm{C}_{1}} \sin 4 \beta}{\mathrm{R}}
\end{aligned}
$$

As was done earlier, we may derive exact expressions for the stresses as

$$
\begin{aligned}
& \sigma_{x x}=\frac{2 p \mu \ddot{B}_{1}}{c_{2} R}\left\{\left[\cos f_{1}^{\prime} x-\cos f_{3}^{\prime} x\right] \cos ^{2} 2 \beta \sin 2 \beta \sin (p t+g y)\right. \\
& +\left[\frac{c_{2}^{2}}{c_{1}^{2}} \sin 2 \beta \sin 2 \alpha \sin f_{1}^{\prime} x+\cos ^{2} 2 \beta \sin f_{3}^{\prime} x\right] \sin 2 \beta \cos (p t+g y) \\
& \sigma_{y y}=\frac{2 p \mu B_{1}}{c_{2} R}\left(\begin{array}{l}
c_{2}^{2} \\
c_{1}^{2}
\end{array}\right)=i \omega 2 \beta\left\{\left(\begin{array}{lll}
c_{1}^{2} \\
\frac{1}{2} \\
c_{2}^{2}
\end{array}{ }^{2} 2 \beta \text { cos } f_{1}^{\gamma}\right.\right. \\
& \left.+\left(\frac{c_{1}^{2}}{c_{2}^{2}}-1-\cos 2 \alpha\right) \cos 2 \beta \cos \mathrm{f}_{3}^{\prime} x\right] \sin (p t+g y) \\
& \left.+\left[\sin 2 \alpha \sin 2 \beta \sin f_{1}^{\prime} x-\left(\frac{c_{1}^{2}}{c_{2}^{2}}-1-\cos 2 \alpha\right) \cos 2 \beta \sin f_{3}^{\prime} x\right] \cos (p t+g y)\right\}
\end{aligned}
$$




$$
\begin{aligned}
\sigma_{z z}= & \frac{2 B_{1} p \lambda}{c_{1} R}\left(\frac{c_{2}}{c_{1}}\right) \sin 2 \beta \cos 2 \beta\left[\sin f_{3}^{\prime} x \cos (p t+g y)-\cos f_{3}^{\prime} x \sin (p t+g y)\right] \\
\sigma_{x y}= & \frac{2 B_{1} p \mu}{c_{2} R}\left(\frac{c_{2}^{2}}{c_{1}^{2}}\right)\left\{\left(-\cos f_{1}^{\prime} x+\cos f_{3}^{\prime} x\right) \sin 2 \alpha \sin 2 \beta \cos 2 \beta \sin (p t+g y)\right. \\
& \left.-\left[\frac{c_{1}^{2}}{c_{2}^{2}} \cos _{2}^{2} 2 \beta \sin f_{1}^{\prime} x+\sin 2 \beta \sin 2 \alpha \sin f_{3}^{\prime} x\right] \cos 2 \beta \cos (p t+g y)\right\}
\end{aligned}
$$

As before for small values of $x$, there are

$$
\begin{aligned}
& \sigma_{x x}=p^{2} \rho x B_{1}\left[\frac{2}{R} \frac{c_{2}}{c_{1}} \sin 2 \beta \cos \alpha\right] \cos p t \quad=p^{2} \rho x B_{1} S_{x x} \cos p t \\
& \sigma_{y y}=p p c_{1} B_{1}\left[-\frac{4}{R} \frac{c_{2}}{c_{1}} \sin 2 \beta \cos 2 \beta\left\{1-\left(\frac{c_{2}}{c_{1}}\right)^{2}\right\}\right] \sin p t \quad=p \rho c_{1} B_{1} S_{y y} \sin p t \\
& \sigma_{\mathrm{zz}}=\mathrm{ppc}_{1} \mathrm{~B}_{1}\left[-\frac{2}{\mathrm{R}}\left\{1-2\left(\frac{\mathrm{c}_{2}}{\mathrm{c}_{1}}\right)^{2}\right\} \frac{\mathrm{c}_{2}}{\mathrm{c}_{1}} \sin 2 \beta \cos 2 \beta\right] \sin \mathrm{pt} \quad=\mathrm{poc}_{1} \mathrm{~B}_{1} \mathrm{~S}_{\mathrm{zz}} \sin \mathrm{pt} \\
& \sigma_{x y}=p^{2} \rho x B_{1}\left[-\frac{2}{R}\left\{\cos ^{2} 2 \beta+\left(\frac{c_{2}}{c_{1}}\right)^{4} \sin ^{2} 2 \alpha(\cos 2 \beta \cos \beta] \cos p t\right.\right. \\
& =p^{2} \rho x B_{1} S_{x y} \cos p t
\end{aligned}
$$

We have also derived formulae for the strains for small $x$ as

$$
\begin{array}{ll}
\epsilon_{\mathrm{xx}}=\frac{\mathrm{pB}_{1}}{\mathrm{c}_{2}}\left[\frac{1}{R}\left\{1-2\left(\frac{\mathrm{c}_{2}}{\mathrm{c}_{1}}\right)^{2}\right\} \sin 2 \beta \cos 2 \beta\right] \sin \mathrm{pt} & =\frac{\mathrm{pB}_{1}}{\mathrm{c}_{2}} \mathrm{E}_{\mathbf{x x}} \sin \mathrm{pt} \\
\epsilon_{\mathrm{yy}}=\frac{\mathrm{pB}}{\mathrm{c}_{2}}\left[\frac{1}{\mathrm{R}} \sin 2 \beta \cos 2 \beta\right] \sin \mathrm{pt} & =\frac{\mathrm{pB}_{1}}{\mathrm{c}_{2}} \mathrm{E}_{\mathrm{yy}} \sin \mathrm{pt}
\end{array}
$$




$$
\epsilon_{\mathrm{xy}}=\frac{\mathrm{pxB}_{1}}{\mathrm{c}_{2}^{2}}\left[-\frac{2}{\mathrm{R}}(\cos 2 \beta \cos \beta)\left\{4\left(\frac{\mathrm{c}_{2}^{2}}{c_{1}^{2}}-1\right) \sin \beta+1\right\}\right] \cos \mathrm{pt}=\frac{\mathrm{pxB}_{1}}{\mathrm{c}_{2}^{2}} \mathrm{E}_{\mathrm{xy}} \cos \mathrm{pt}
$$

The final case treated is that of the shear waves which are incident at an angle such that no real reflected longitudinal wave is possible. This occurs wherever $\beta$ is large enough that for the Poisson's ratio of the material,

$$
\sin \beta>\frac{c_{2}}{c_{1}}=\sqrt{\frac{1-2 \nu}{2-2 \nu}}
$$

The treatment of this case is more complicated although the same condition of zero stress $\sigma_{x x}$ or $\sigma_{\mathrm{yx}}$ on the free surface is the basis for the solution. Following Kolsky's notation we again express the incident shear wave as

$$
\psi_{1}=\operatorname{Re}\left\{B_{1} e^{\left[i\left(p t+f i x+g_{1}^{\prime} y\right)\right]}\right\}
$$

where

$$
\begin{aligned}
& f_{1}^{\prime}=p \cos \beta / c_{2} \\
& g_{1}^{\prime}=p \sin \beta / c_{2} .
\end{aligned}
$$

The reflected shear wave is

$$
\psi_{2}=R_{\theta}\left\{B_{2} \cdot\left[i\left(p t-f_{2}^{\prime} x+g_{2}^{\prime} y+\delta_{1}\right)\right]\right\}
$$

a second resulting wave is

$$
\psi_{3}=\operatorname{Re}\left\{B_{3} e^{\left[i\left(p t-f_{3}^{\prime} x+g_{3}^{\prime} y+\delta_{2}\right)\right]}\right\}
$$

where

$$
\begin{aligned}
& f_{2}^{\prime}=p \cos \beta / c_{2} \text { and } g_{2}^{\prime}=p \sin \beta / c_{2} \\
& f_{3}^{\prime}=p(\cos \alpha) / c_{1} \text { and } g_{3}^{\prime}=p(\sin \alpha) / c_{1} .
\end{aligned}
$$


Here $\sin \alpha$ is not real but complex and everywhere must be replaced by $\left[\left(c_{1} / c_{2}\right) \sin \beta\right]$. Also $\cos$ $\alpha$ is imaginary and

$$
(\cos \alpha)^{2}=1-\left(\frac{c_{1}}{c_{2}}\right)^{2}(\sin \beta)^{2}<0 .
$$

In this case the two phase angles $\delta_{1}$ and $\delta_{2}$ are non-zero.

The second wave in this case is a surface wave described by

$$
\psi_{3}=B_{3} e^{-m x} \cos \left(p t+g_{3}^{t} y+\delta_{2}\right)
$$

where

$$
\begin{aligned}
& \mathrm{m}=\left(\frac{\mathrm{p}}{\mathrm{c}_{1}}\right)\left[\left(\frac{\mathrm{c}_{1}}{\mathrm{c}_{2}}\right)^{2} \sin ^{2} \beta-1\right]^{1 / 2} \\
& \mathrm{~g}_{3}^{\prime}=\frac{\mathrm{p}(\sin \alpha)}{\mathrm{c}_{1}}=\mathrm{p} \frac{(\sin \beta)}{\mathrm{c}_{2}}=\mathrm{g}_{1}^{\prime}=\mathrm{g}_{2}^{\prime}=\mathrm{g} .
\end{aligned}
$$

Note that physical considerations require that the exponential exponent be negative.

The values of the variables are

$$
\begin{aligned}
& \sin \alpha=\frac{c_{1}}{c_{2}} \sin \beta \\
& \cos \alpha=-i \sqrt{\left(\frac{c_{1}}{c_{2}}\right)^{2}(\sin \beta)^{2}-1} \\
& \mathrm{~B}_{2}=\mathrm{B}_{1} \\
& \frac{\mathrm{B}_{3}}{\mathrm{~B}_{1}}=\frac{2 \frac{\mathrm{C}_{2}}{\mathrm{C}_{1}} \sin 2 \beta \cos 2 \beta}{\mathrm{R}^{*}}=\frac{\frac{\mathrm{C}_{2}}{\mathrm{c}_{1}} \sin 4 \beta}{\mathrm{R}^{*}} \\
& \delta_{2}=\sin ^{-1}\left\{\frac{2}{R_{*}^{*}} \frac{c_{2}}{c_{1}} \sin \beta \sin 2 \beta \sqrt{\left(\frac{c_{1}}{c_{2}} \sin \beta\right)^{2}-1}\right\}
\end{aligned}
$$




$$
\begin{aligned}
& \delta_{1}=2 \delta_{2} \\
& R *=\left(\cos ^{4} 2 \beta+4\left(\frac{c_{2}}{c_{1}}\right)^{2} \sin ^{2} \beta \sin ^{2} 2 \beta\left(\left(\frac{c_{1}}{c_{2}}\right)^{2} \sin ^{2} \beta-1\right)^{1 / 2}\right.
\end{aligned}
$$

The values of $B_{2} / B_{1}$ and $B_{3} / B_{1}$ for the incident shear wave are shown in Figure 6 and in Table II for various Poisson's ratios. The values of $\delta_{1}$ and $\delta_{2}$ are shown in Table II.

The exact values for the stresses are

$$
\begin{aligned}
& \sigma_{x x}=\frac{p_{1} \mu}{c_{2}}\left\{2 \sin 2 \beta \cos \left(f_{1}^{\prime} x-\delta_{2}\right)\right. \\
& \left.-\frac{B_{3}}{B_{1}} \frac{c_{1}}{c_{2}} \cos 2 \beta e^{-\frac{p x}{c_{1}} \sqrt{\left(\frac{c_{1}}{c_{2}} \sin \beta\right)^{2}-1}}\right\} \sin \left(p t+g y+\delta_{2}\right) \\
& \sigma_{y y}=-\frac{\mathrm{pB}_{1} \mu}{\mathrm{c}_{2}}\left\{2 \sin 2 \mathrm{~B} \cos \left(f_{1}^{\prime} \mathrm{x}-\delta_{2}\right)+\frac{\mathrm{B}_{3}}{\mathrm{~B}_{1}} \frac{\mathrm{c}_{2}}{\mathrm{c}_{1}}\left[2\left(\frac{\mathrm{c}_{1}^{2}}{\mathrm{c}_{2}^{2}}-1\right)\right. \text {. }\right. \\
& \left.\left.-\frac{c_{1}^{2}}{c_{2}^{2}} \cos 2 \beta\right] e^{-\frac{p x}{c_{1}} \sqrt{\left(\frac{c_{1}}{c_{2}} \sin \beta\right)^{2}-1}}\right\} \sin \left(p t+g y+\delta_{2}\right) \\
& \sigma_{z z}=-\frac{p}{c_{1}}\left(\frac{c_{1}^{2}}{c_{2}^{2}}-2\right) \mu B_{1}\left(\frac{B_{3}}{B_{1}}\right) e^{\left.-\frac{p x}{c_{1}} \sqrt{\left(\frac{c_{1}}{c_{2}} \sin B\right.}\right)^{2}-1} \sin \left(p t+g y+\delta_{2}\right) \\
& \sigma_{x y}=-\frac{2 p \underline{B}_{1} H}{c_{2}}\left\{\cos 2 \beta \sin \left(f_{1}^{\prime} x-\delta_{2}\right)\right. \\
& \left.+\frac{B_{3}}{B_{1}} \sin B \sqrt{\left(\frac{c_{1}}{c_{2}} \sin B\right)^{2}-1} e^{-\frac{p x}{c_{1}} \sqrt{\left(\frac{c_{1}}{c_{2}} \sin B\right)^{2}-1}}\right\} \cos \left(p t+g y+\sigma_{2}\right)
\end{aligned}
$$

22 
As before, we have derived approximate values for the stress for small values of $\mathrm{x}$ as

$$
\begin{aligned}
& \sigma_{\mathrm{xX}}=\mathrm{p}^{2} \rho \mathrm{xB}\left[\frac{2}{\mathrm{R*}}\left(\frac{\mathrm{c}_{2}}{\mathrm{C}_{1}}\right) \sin 2 \beta \sqrt{\left(\frac{\mathrm{c}_{1}}{\mathrm{c}_{2}} \sin \beta\right)^{2}-1}\right]\left[\sin \left(\mathrm{pt}+\delta_{2}\right)\right]=\mathrm{p}^{2} \rho \times \mathrm{B}_{1} \mathrm{~S}_{\mathrm{XXX}} \sin \left(\mathrm{pt}+\delta_{2}\right) \\
& \sigma_{\mathrm{yy}}=p \rho c_{2} \mathrm{~B}_{1}\left[-\frac{4}{\mathrm{R}^{\star}}\left(1-\left(\frac{\mathrm{c}_{2}}{\mathrm{c}_{1}}\right)^{2}\right) \sin 2 \beta \cos 2 \beta\right] \sin \left(\mathrm{pt}+\delta_{2}\right) \quad=p \rho \mathrm{c}_{2} \mathrm{~B}_{1} \mathrm{~s}_{\mathrm{yy}} \sin \left(\mathrm{pt}+\sigma_{2}\right) \\
& \sigma_{z z}=p \rho c_{2} B_{1}\left[-\frac{2}{R^{*}}\left(1-2\left(\frac{c_{2}}{c_{1}}\right)^{2}\right) \sin 2 \beta \cos 2 \beta\right] \sin \left(p t+\delta_{2}\right) \cdot=p \rho c_{2} B_{1} S_{z z} \sin \left(p t+\delta_{2}\right) \\
& \sigma_{x y}=p^{2} \rho x B_{1}\left[-\frac{2}{R^{*}} \cos 2 \beta \cos \beta\left\{4\left(\frac{c_{2}^{2}}{c_{1}^{2}}-1\right) \sin ^{2} \beta+1\right\}\right] \cos \left(p t+\sigma_{2}\right) \\
& =p^{2} \rho x B_{1} S_{x y} \cos \left(p t+\delta_{2}\right)
\end{aligned}
$$

The values of the numerical coefficient (S and E) are plotted in Figure 7 and given in Table II. Upon examination, it may be noted that the coefficients for the stresses are essentially of the same form regardless of whether there is a reflected longitudinal wave ( $\beta<$ the critical angle) or a surface wave $(\beta>$ the critical angle).

Finally it may be shown that the equations for the strains when $\beta$ is greater than the critical angle are identical to those previously given when $\beta$ is less than the critical angle with the exception that $R$ * must be substituted for $R$ when the surface wave is generated. The values for the coefficients for various Poisson's ratios are shown in Figure 8 and given in Table II.

It is hoped that at some future date the information incorporated in this paper can be used to calculate the maximum stress which results from both incoming transverse and shear waves. 


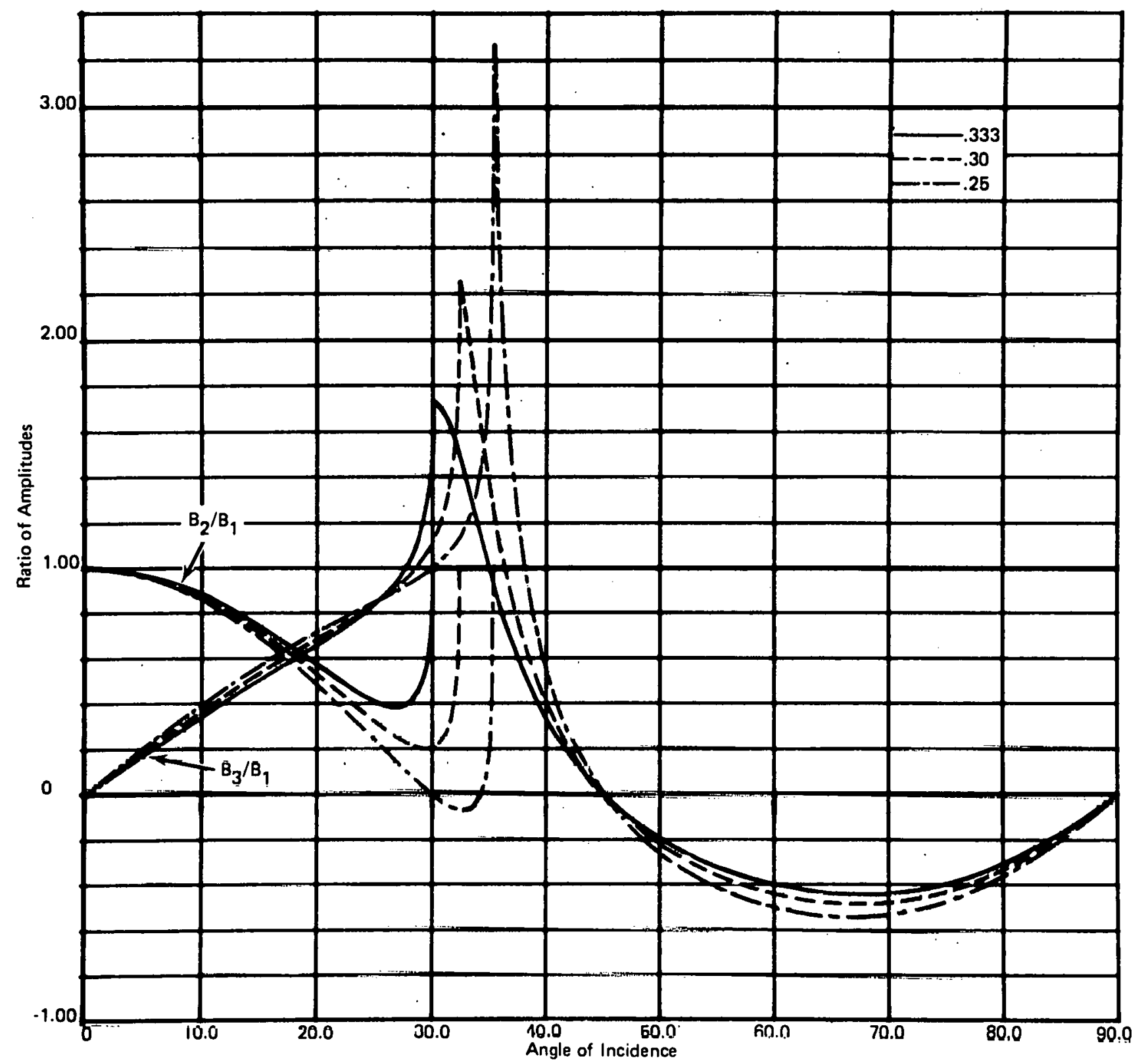

Figure 6. Plot of Ratios of Amplitude $B_{2} / B_{1}$ and $B_{3} / B_{1}$ vs.

Angle of Incidence for Poisson's Ratios of 0.25 , 0.30 and 0.333 . 


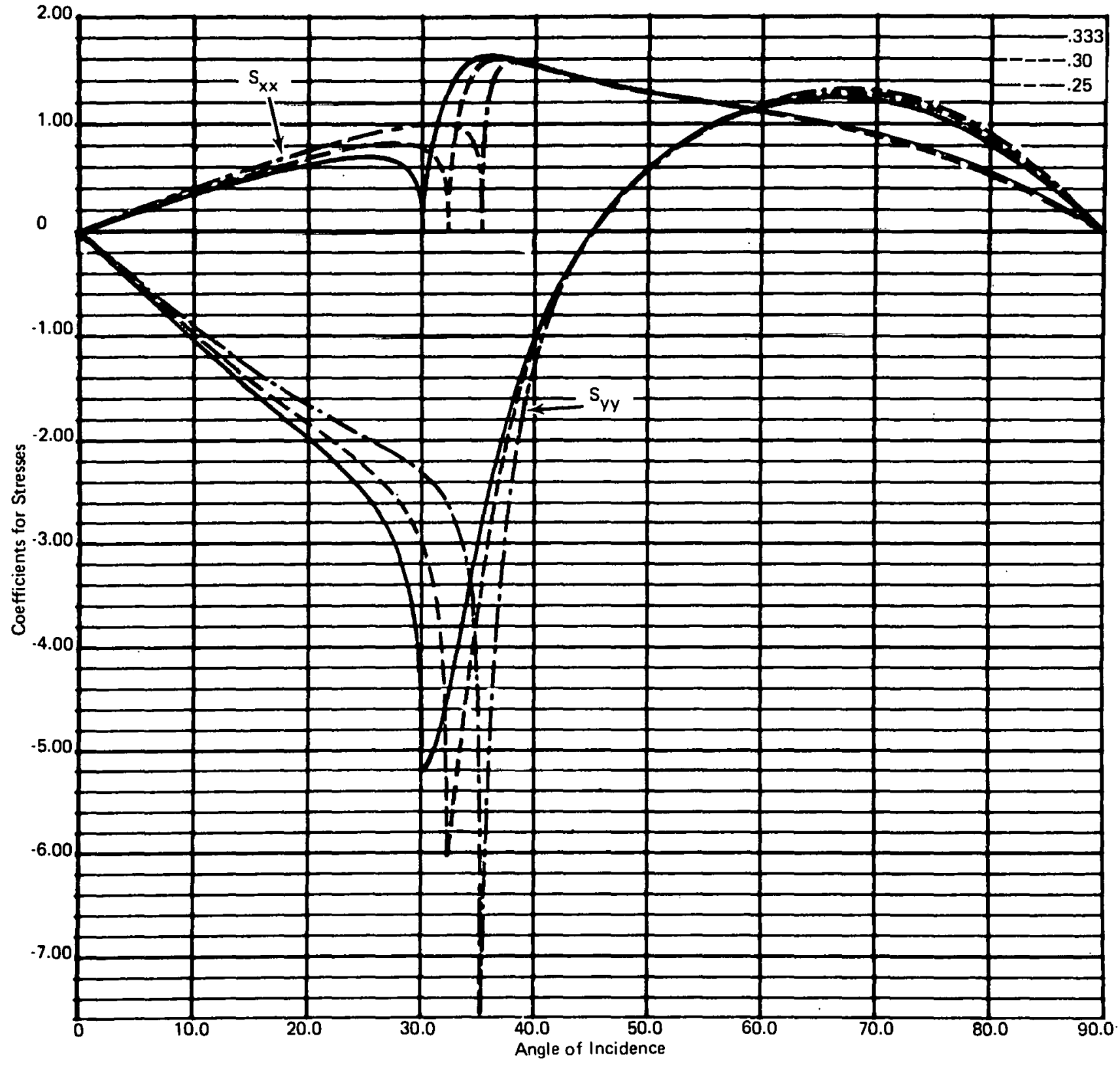

Figure 7a. Plot of the Coefficients $S_{x x}$ and $S_{y y}$ vs. Angle of Incidence for Poisson!s Ratios of $0.25,0.30$ and 0.333 , Ineident Shear Wave. 


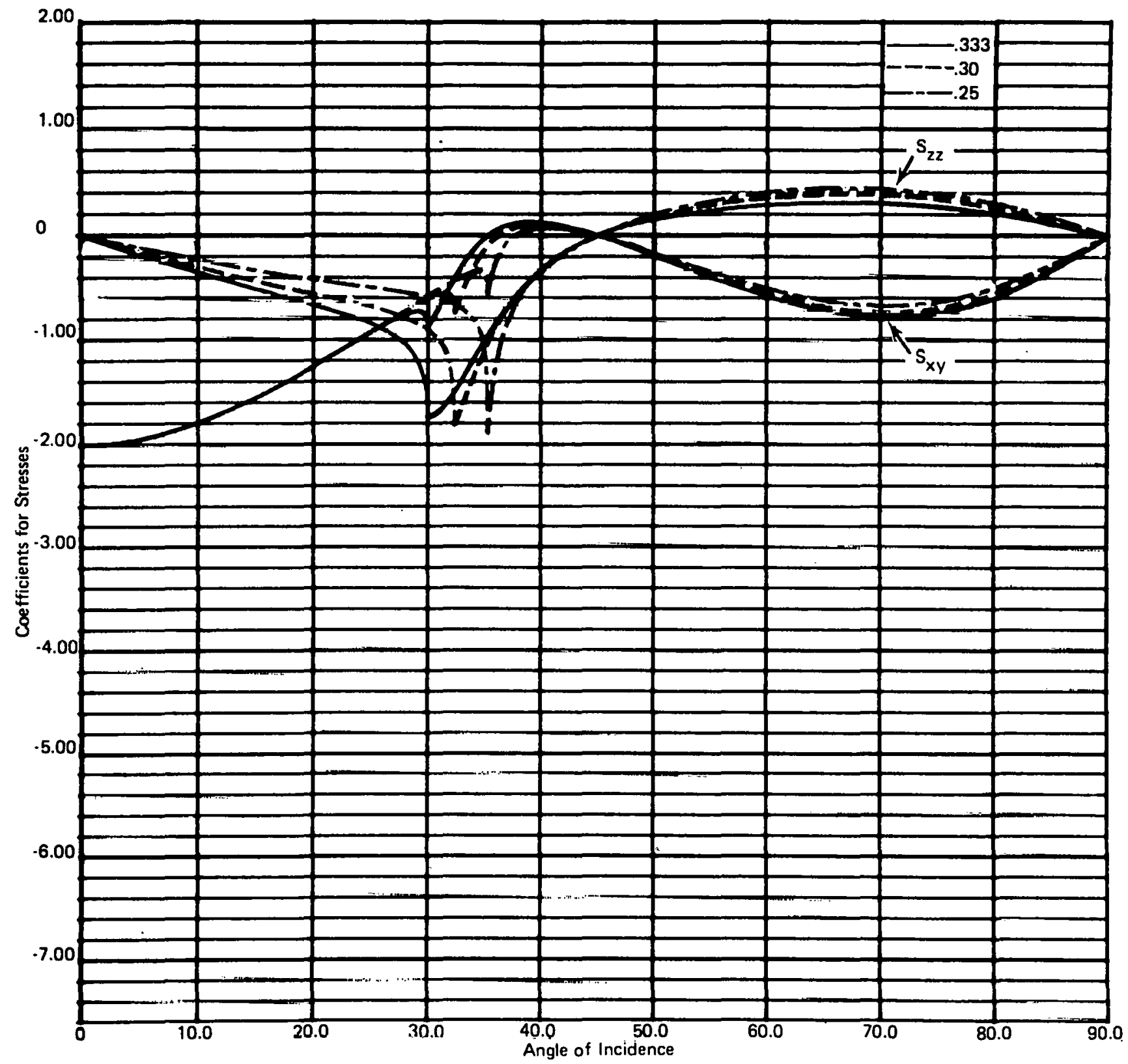

Figure 7b. Plot of the Coefficients $S_{x y}$ and $S_{z z}$ vs. Angle of Incidence for Poisson's Ratios of $0.25,6.30$ and 0.333 , Incident Shear Wave. 


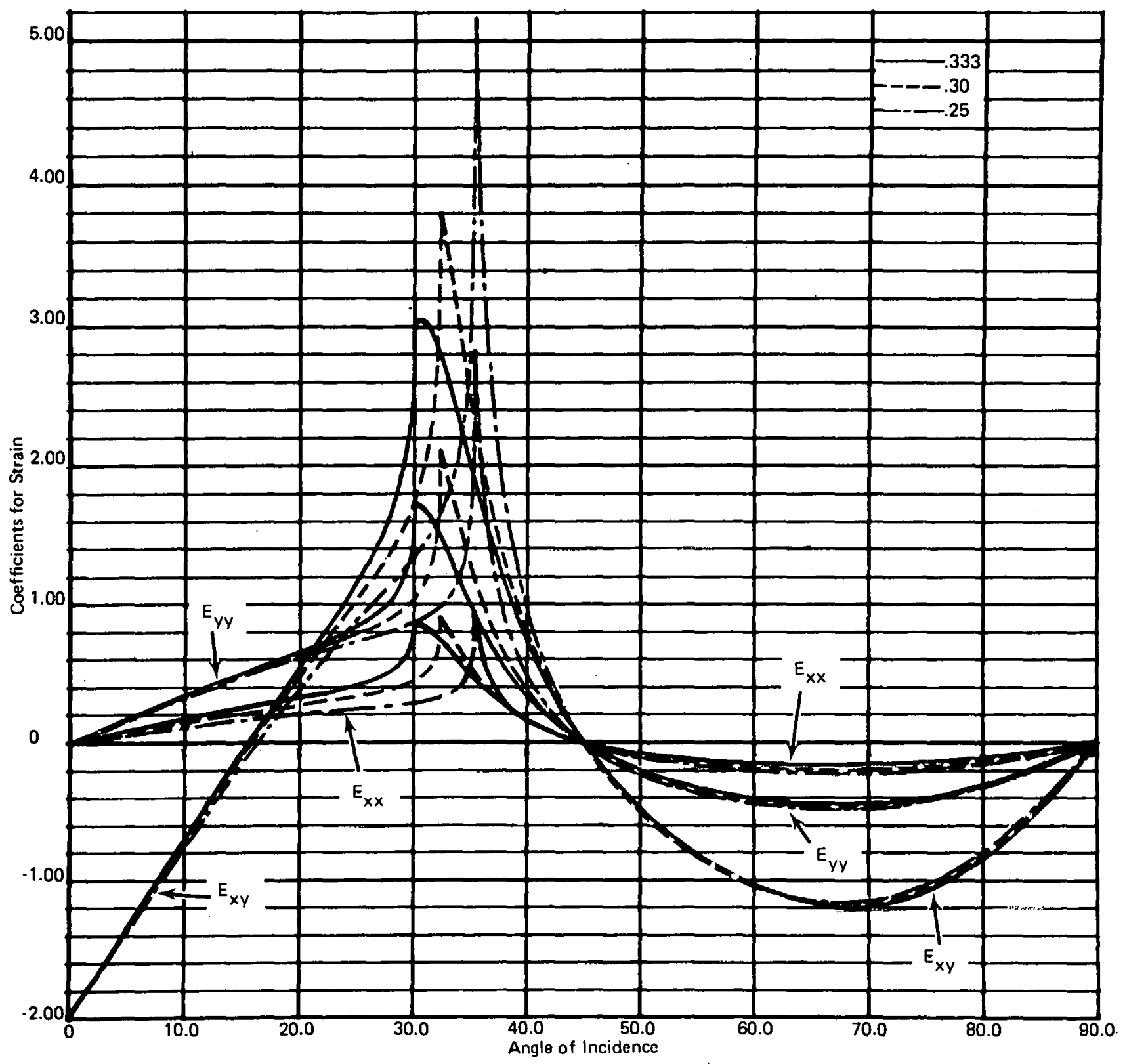

Figure 8. Plot of the Coefficients $E_{x x}, E_{y y}$ and $E_{x y}$ vs. Angle of Incidence for Poisson's Ratios of $0.25,0.30$ and 0.333 , Incident Shear Wave. 
Values of Amplitudes, Stresses and Strains for an Incoming Shear Wave, Pois son's Ratio of 0.25

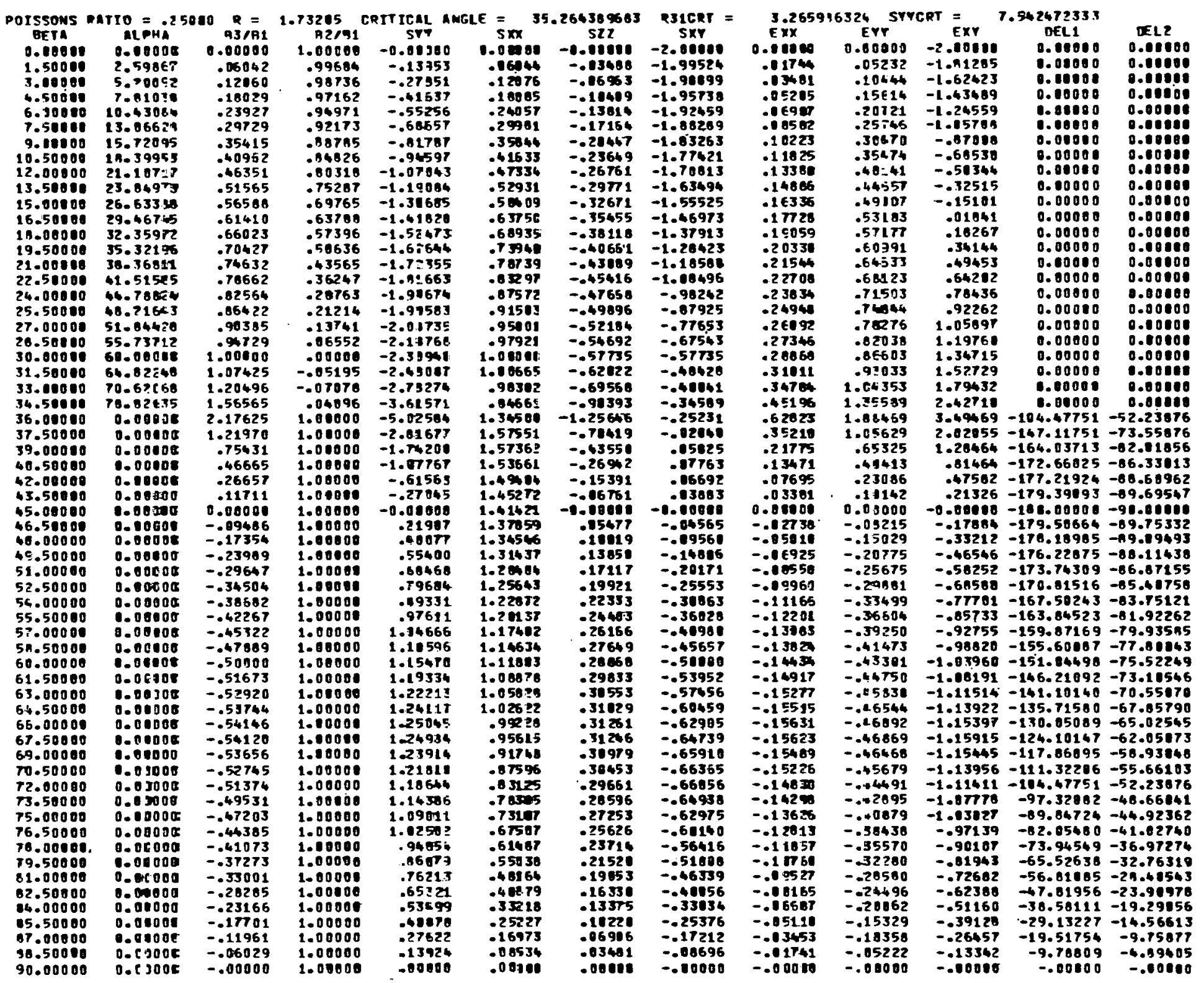


TABLE IIb

Valies of Amplitudes, Stresses and Strains for an Incoming Shear Wave, Poisson's Ratio of 0.30

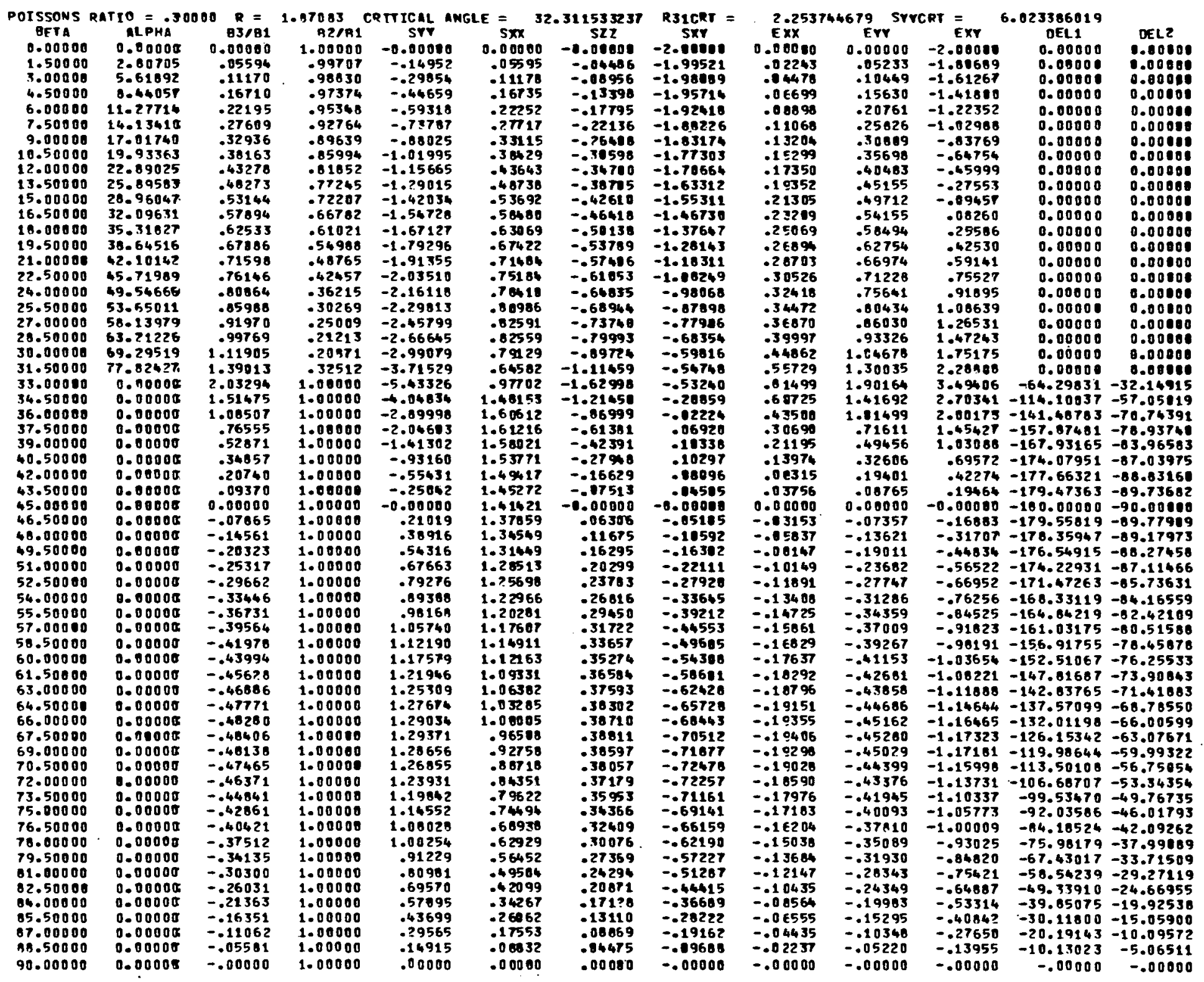


TABLE IIC

Values of Amplitıdes, Stresses and Strains for an Incoming Shear Wave, Poisson's Ratio of 0.333

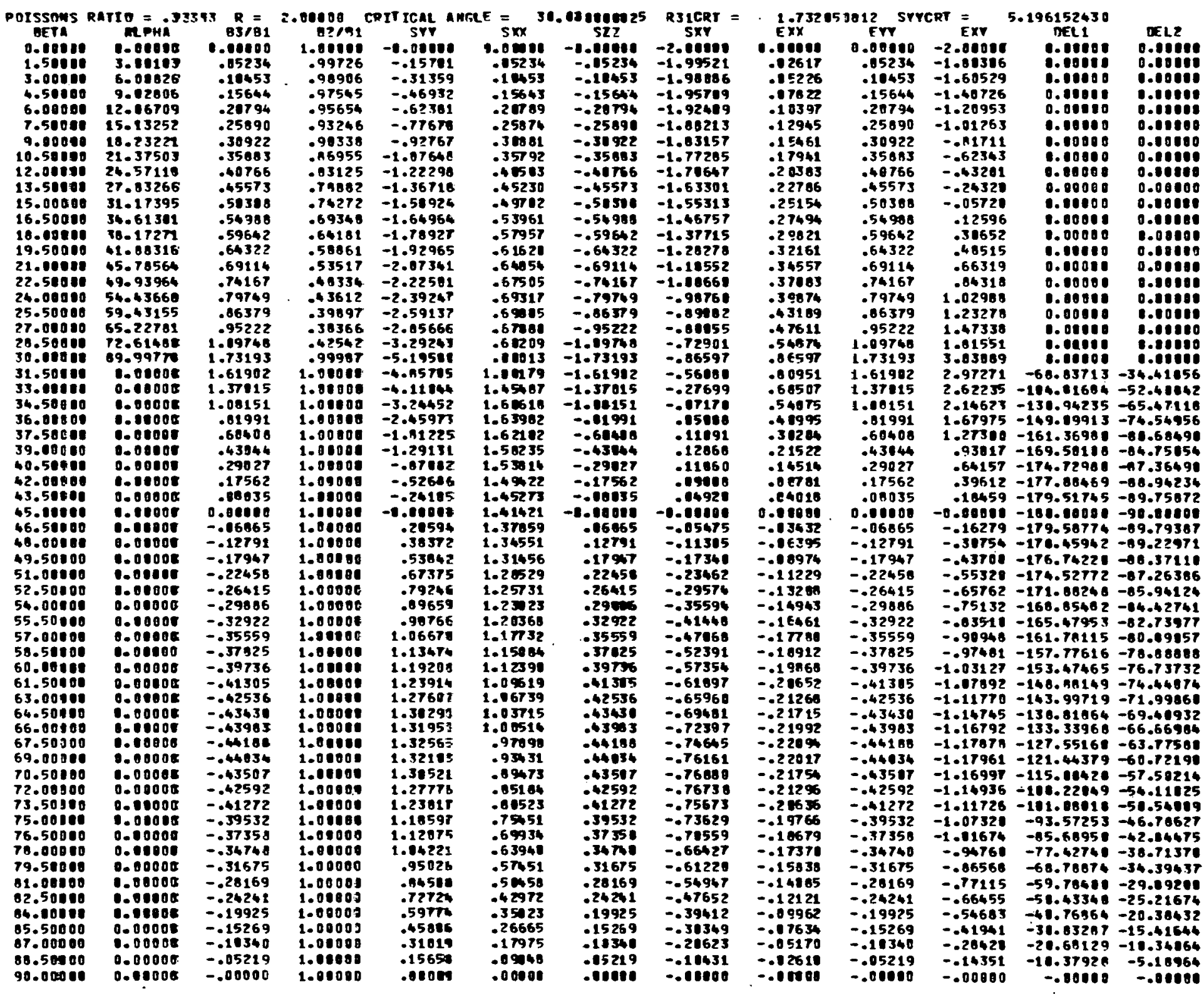


References

1. Kolsky, H., Stress Waves in Solids, Dover, NY, 1963, pp. 24-38.

2. Mason, Warren P., Physical Acoustics and the Properties of Solids, D. Van Nostrand and Company, Princeton, NJ, pp. 22-27, 1958.

3. Båth, Markus, Mathematical Aspects of Seismology, Elsevier Publishing Company, Amsterdam, 1968, pp. 255-259.

4. Knopoff, L., R. W. Fredriks, A. F. Gangi and L. D. Porter, "Surface Amplitudes of Reflected Body Waves." Geophysics, Vol XXI, No. 4 (Oct, 1957), pp. 842-847. 
DISTRIBUTION:

Systems, Science and Software

P. O. Box 1620

La Jolla, CA 92038

Attn: D. R. Dismuke

US Department of Energy

Nevada Operations Office

P.O. Box 14100

Las Vegas, NV 89114

Attn: P. N. Halstead

US Geological Survey

Mail Stop 966

P.O. Box 25046

nenver Federal Center

Denver, CO 80225

Attn: W. W. Hays

Computer Sciences Corporation

Systems Division

6565 Arlington Boulevard

Falls Church, VA 22046

Attn: J. A. Lahoud

University of California

Lawrence Livermore Laboratory

P.O. Box 808

Livermore, CA 94550

Attn: M. D. Nordyke, L 49

Los Alamos Scientific Laboratory

P.O. Box 1663

Los Alamos, NM 87545

Attn: K. A. Olsen, G-2

.T. A. Blume \& Assoclates, Engineers Sheraton-Palace Hotel

100 Jessie Street

San Francisco, CA 94105

Attn: R. E. Scholl

University of California

Dept. of Civil Engineering

477. Avis Hall

Berkeley,' CA 94720

Attn: H. B. Seed

Ramea \& Moorc

500 Sansome Street

San Francisco, CA 04111

Attn: L, Winters
1000

1100

- 1101

1110

1111

1111

1111

$1111 \mathrm{~A}$

$1111 \mathrm{~A}$

$1111 \mathrm{~A}$

1120

1123

1124

1130

1281

1355

1710

1730

1750

$1760^{\circ}$

5300

5310

5330

5340

5341

5341

5431

5433

9324

8266

3141

3151

DOE/TIC (25)

G. A. Fowler

C. D. Broyles

J. R. Banister (5)

J. D. Kennedy

C. R. Mehl (2)

R. C. Bass

L. J. Vortman

F. F. Dean (5)

H. D. Garbin

D. M. Tendall

G. E. Hansche

H. R. Holmes

J. W: Allen

II. E. Vinoy

S. W. Key

P. A. Stokes

V. E. Blake

C. H. Mauney

J. E. Stiegler

J. Jacobs

O. E. Jories

W. D. Weart

R. W. Lynch

M. L. Kramm

L. W. Scully

D. M. Ellett (5)

W. A. Von Riesemann

J. M. Freedman

F. D. Gutierrez

E. A. Aas

T. Werner (5)

W. L. Garner (3)

For DOE/TIC (Unlimited Release)

(R. P. Campboll, 317?:=3) 